\title{
Optical Design and Optimization of a Micro Zoom System with Liquid Lenses
}

\author{
Wei Zhang*, Dan Li, and Xin Guo \\ Engineering Research Center of Optical Instrument and System, Ministry of Education, Shanghai Key \\ Laboratory of Modern Optical System, School of Optical-Electrical and Computer Engineering, University \\ of Shanghai for Science and Technology, Shanghai 200093, China
}

(Received June 5, 2013 : revised August 26, 2013 : accepted September 3, 2013)

\begin{abstract}
A micro zoom system without moving elements by use of two liquid lenses is designed and optimized in this paper. The zoom equations of the system composed of two liquid lenses are deduced. The structure parameters including radius and thickness of a conical double-liquid electrowetting based lens are analyzed and calculated. Because the liquid thickness varies non-linearly with the radius of the interface, it's very difficult to optimize a real liquid lens using commercial optical design software directly. Through the Application Programming Interface (API) of the optical design software CODE V, a zoom system with two real electrowetting based liquid lenses is modeled and optimized. A two-liquid-lens zoom system without moving elements, with a zoom factor of 1.8 and a compact structure of $10 \mathrm{~mm}$ is designed for illustration. This can be useful for the camera design of mobile phones, tablets and so on. And this paper presents a convenient way of designing and optimizing a zoom system including liquid lenses by commercial optical design software.
\end{abstract}

Keywords: Optical design, Liquid lens, Micro zoom system, No moving elements

OCIS codes : (220.0220) Optical design and fabrication; (220.4830) System design; (230.4685) Optical microelectromechanical devices

\section{INTRODUCTION}

Miniature zoom systems are widely used for many advanced optical systems today. The conventional zoom system always has more than two elements with nonlinear movement to implement focus variation and imaging shift compensation at the same time. This will lead to large volume and complicated structure of the system. The liquid lens is a novel type of optical device. With the character of active zooming, the liquid lens can make a zoom optical system more miniature, and has very wide potential applications in mobile phones, micro projectors and so on.

There are three main types of tunable-focus liquid lenses: liquid crystal lens [1-2], electrowetting effect based liquid lens [3-5] and liquid-filled membrane lens [6-8]. The electrowetting effect based liquid lens was first reported by the researchers of Philips Optical in 2003 in [3]. The focal length can be adjusted under different external voltages by changing the curvature of the interface between two kinds of immiscible liquids. This kind of liquid lens has short response time and wide focus tunable range. Now Varioptic can provide a series of commercial products of this kind of liquid lens.

By use of the liquid lens, zoom optical systems can be designed without moving elements. This will avoid many disadvantages of conventional zoom systems, which may be complicated, fragile and expensive. Some researchers published results and analysis of zoom optical systems with variable power liquid lenses. Kupier [4] pointed out that more than two liquid lenses are required to implement a zoom system. Zhang [9] analyzed the principle of the two liquid lenses zoom system by Gaussian optics. The paraxial and third order aberration and first order chromatic aberration analysis of zoom systems based on liquid lenses were studied in [10]. Sun, Park and Fang [11-13] gave examples of the zoom system design with a liquid lens and one group of moving lenses. But the zoom system with no moving elements has not been discussed. And much research has been reported to try to design zoom systems with liquid lenses in [14-16]. However there is

\footnotetext{
*Corresponding author: zwopxa@gmail.com

Color versions of one or more of the figures in this paper are available online.
} 
still not a comprehensive method to set up a zoom system with liquid lenses, nor a convenient way to optimize the system by using commercial optical design software.

In this paper, we focus on the similarity of the zoom system with liquid lenses. The zoom equations of the system composed of two liquid lenses are deduced. The characters of the structure parameters of the conical doubleliquid electrowetting based lens are analyzed and calculated by Matlab software during the focal length change. Through the Application Programming Interface (API) of the commercial optical design software CODE $\mathrm{V}$, a real liquid lens model is set up and a zoom system with liquid lenses is optimized successfully. This will make the design of the zoom system with liquid lenses much more convenient. Then a two-liquid-lenses zoom system without moving elements is designed for illustration, which has a zoom factor of 1.8 and a compact structure of $10 \mathrm{~mm}$. This may be useful for a mobile phone camera, a tablet camera and so on.

\section{ZOOM EQUATION OF TWO-LIQUID-LENS VARIABLE FOCUS SYSTEM}

The variable focus system must satisfy two basic conditions, which are: adjustable focal length, and fixed image plane. To satisfy the two conditions at the same time, there need to be more than two pieces of liquid lens. One is for focal length tuning and the other is for imaging shift compensation.

A two-liquid-lens zoom system is shown in figure 1. In the system, the liquid lens 1 (LL1) is for changing the focal length; while the liquid lens 2 (LL2) is for compensating the imaging shift.

In Fig. 1, the system is composed of only two pieces of liquid lens. $l_{1}$ is the distance between the object plane and LL1, $l_{1}^{\prime}$ is the image distance of the LL1, $l_{2}$ is the object distance of the LL2, $l_{2}{ }^{\prime}$ is the distance between LL2 and the imaging plane, and $L_{12}$ is the distance between the two liquid lenses.

Since the conjugate distance between the object plane and the imaging plane of the system is fixed, the $l_{1}, L_{12}$ and $l_{2}$ 'shown in Fig. 1 are constants. When the focal length of LL1 (presented by $f_{1}^{\prime}$ ) is varied, the $l_{1}^{\prime}$ will be changed correspondingly. Supposing the shifting of $f_{1}^{\prime}$ is very small, the differential function of LL1 can be described as equation (1) according to the Gaussian Optics theory:

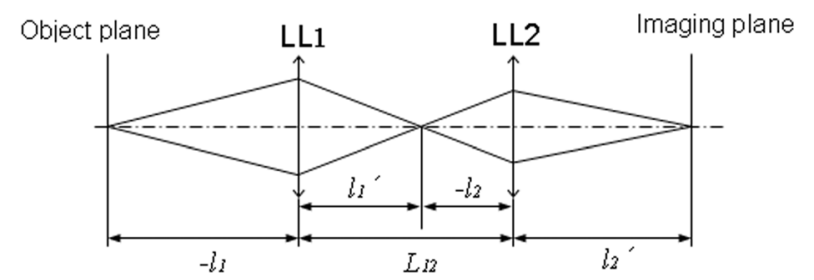

FIG. 1. Schematic of two-liquid-lens zoom system.

$$
d l_{1}{ }^{\prime}=\frac{l_{1}{ }^{2}}{\left(l_{1}+f_{1}{ }^{\prime}\right)^{2}} d f_{1}{ }^{\prime}
$$

According to the geometric relationship, $l_{1}{ }^{\prime}$ and $l_{2}$ should satisfy the equation: $l_{2}=l_{1}^{\prime}-L_{12}$.

Since $L_{12}$ is a constant, we get:

$$
d l_{2}=d l_{1}^{\prime}
$$

Presuming the focal length of LL2 (presented by $f_{2}^{\prime}$ ) is fixed, the image distance $l_{2 a}{ }^{\prime}$ will be altered with the shifting of $l_{2}$, which is described as equation (3):

$$
d l_{2 a}{ }^{\prime}=\frac{f_{2}{ }^{2}}{\left(l_{2}+f_{2}^{\prime}\right)^{2}} d l_{2}
$$

On the other hand, presuming $l_{2}$ is fixed and $f_{2}{ }^{\prime}$ is a variation, the image distance $l_{2 b}{ }^{\prime}$ will be varied with $f_{2}{ }^{\prime}$. The differential function of $l_{2 b}{ }^{\prime}$ and $f_{2}{ }^{\prime}$ is shown as equation (4):

$$
d l_{2 b}{ }^{\prime}=\frac{l_{2}{ }^{2}}{\left(l_{2}+f_{2}{ }^{\prime}\right)^{2}} d f_{2}^{\prime}
$$

Since the image distance of LL2 (presented by $l_{2}{ }^{\prime}$ ) should be a constant in the system, the shifting of $l_{2}{ }^{\prime}$ caused by the variation of $l_{2}$ should be compensated by the variation of $f_{2}{ }^{\prime}$. That means:

$$
d l_{2 b}{ }^{\prime}=-d l_{2 a}{ }^{\prime}
$$

According to the equations (3), (4), (5), the relationship between $l_{2}$ and $f_{2}{ }^{\prime}$ can be described as:

$$
\frac{d l_{2}}{d f_{2}^{\prime}}=-\frac{l_{2}^{2}}{f_{2}^{\prime 2}}
$$

According to the Gaussian Optics theory, we can get equation (7):

$$
l_{2}=\frac{f_{2}^{\prime} l_{2}^{\prime}}{l_{2}^{\prime}-f_{2}^{\prime}}
$$

According to the equations (1), (2), (6), and (7), the differential function of $f_{1}^{\prime}$ and $f_{2}{ }^{\prime}$ is gotten: 


$$
\frac{l_{2}^{\prime}{ }_{2}}{\left(f_{2}^{\prime}{ }_{2}-l_{2}^{\prime}\right)^{2}} d f_{2}^{\prime}+\frac{l_{1}^{2}}{\left(l_{1}+f_{1}^{\prime}\right)^{2}} d f_{1}^{\prime}=0
$$

Solving the equation (8), we get the general solution:

$$
\frac{l_{2}^{\prime 2}}{f^{\prime}{ }_{2}-l_{2}^{\prime}}+\frac{l_{1}^{2}}{l_{1}+f^{\prime}{ }_{1}}=K
$$

where $K$ is a constant which is independent of $f_{1}^{\prime}$ and $f_{2}{ }^{\prime}$, but only depends on the structure parameters of the system. With the presumptions of $l_{1}=40 \mathrm{~mm}$ and $l_{2}{ }^{\prime}=3$ $\mathrm{mm}$, the curve of the focal length of the two liquid lenses at different $K$ value is calculated and shown in Fig. 2 . When $K$ is increasing, the overall length of the system will diminish.

The relationship between the magnification of the system and $f_{1}^{\prime}$ is given by the formula (10) and is shown in Fig. 3.

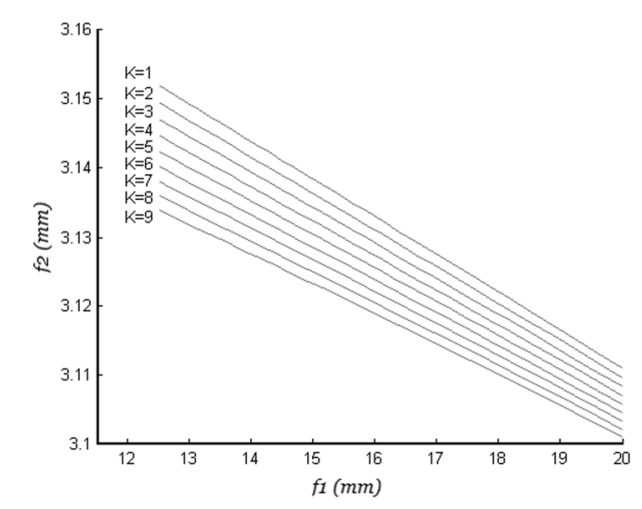

FIG. 2. Curve of the focal length of the two liquid lenses at different $K$ value. The calculation is based on the presumptions of $l_{1}=40 \mathrm{~mm}$ and $l_{2}{ }^{\prime}=3 \mathrm{~mm}$.

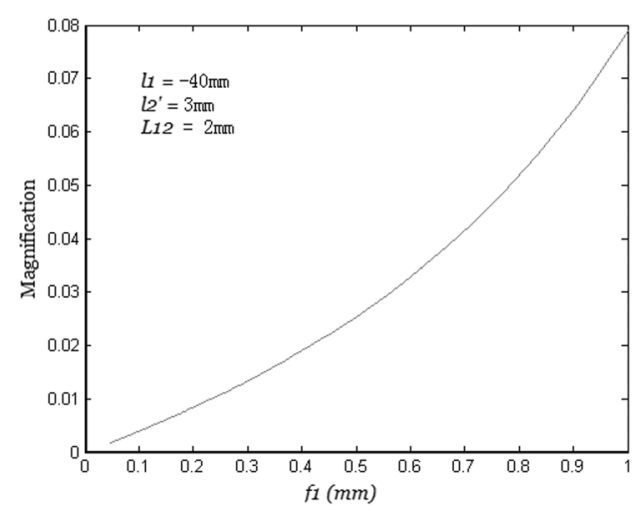

FIG. 3. Curve of the magnification of the system and $f_{l}^{\prime}$. The calculation is based on the presumptions of $l_{1}=40 \mathrm{~mm}, l_{2}{ }^{\prime}=3 \mathrm{~mm}$, and $L_{12}=2 \mathrm{~mm}$.

$$
M=m_{1} m_{2}=\frac{m_{1} l_{2}^{\prime}}{m_{1} l_{1}-L_{12}}=\frac{f_{1}^{\prime} l_{2}^{\prime}}{f_{1}^{\prime}\left(l_{1}-L_{12}\right)-l_{1} L_{12}}
$$

According to the magnification needed and the variable range of the liquid lens, the initial system can be set up by solving the zoom equation.

\section{METHODOLOGY OF OPTICAL DESIGN FOR A VARIABLE FOCUS SYSTEM WITHOUT MOVING ELEMENTS}

\subsection{Structure Parameters of the Conical Double-liquid Electrowetting Based Lens}

Several years ago researchers of Philips Optical announced a kind of electrowetting effect based liquid lens as a varifocal component. This type of liquid lens is composed of a cylindrical cavity and two kinds of immiscible liquid. One is conducting and the other is insulating. The refractive index of the two kinds of liquid is different. The interface of the two immiscible liquids would be perfectly spherical if the density of the two kinds of liquid were the same [15]. The contact angle of these two kinds of liquid and the cavity insulating wall $(\theta$ in Fig. 4) can be influenced by an external electric field for the electrowetting effect. Then the curvature between the two immiscible liquids can be changed and the focal length can be adjusted.

Figure 4 shows a simplified schematic of the electrowetting based liquid lens with conical structure (This structure is applied in the liquid lens of Varioptic). When the curvature between the two immiscible liquids is changed, the position of the summit of the spherical interface will be altered at the same time.

Considering the lens shown in Fig. 4, $n_{1}$ and $n_{2}$ are the refractive indices of liquid 1 and liquid 2 respectively, $d_{02}$ is the thickness of liquid 2 when the interface is plane, $r$ is the radius of curvature of the interface, $d_{2}$ is the thickness of liquid 2 when the radius is changed to $r, \theta$ is the contact angle, $a_{1}$ and $a_{2}$ are the top and bottom radii of the chamber, $a$ is the angle between the generatrix of the cone and the core axis of the chamber.

Presuming the volume of liquid 2 is $V_{p}$ when the interface is plane and $V_{r}$ when the interface is spherical. $V_{a}$ and $V_{b}$

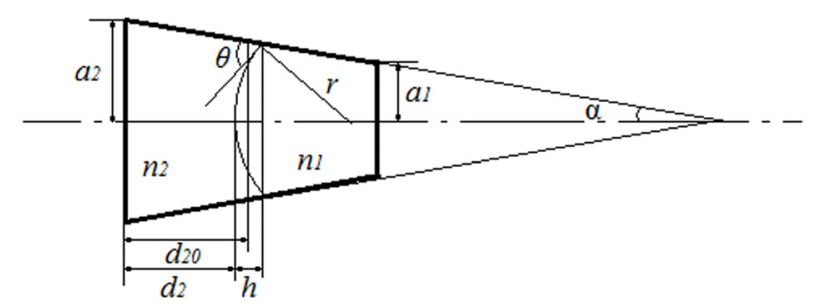

FIG. 4. Schematic of the electrowetting based liquid lens with conical structure. 
can be respectively described as:

$$
\left\{\begin{array}{c}
V_{p}=\frac{\pi a_{2}{ }^{3}}{3 \tan \alpha}-\frac{\pi\left(a_{2}-d_{20} \tan \alpha\right)^{3}}{3 \tan \alpha} \\
V_{r}=\frac{\pi a_{2}{ }^{3}}{3 \tan \alpha}-\frac{\pi(r \cos (\theta-\alpha))^{3}}{3 \tan \alpha}-\frac{\pi h^{2}}{3}(3 r-h)
\end{array}\right.
$$

Where $h=r(1-\sin (\theta-\alpha))$.

Since the volume of each liquid is constant, $V_{p}$ and $V_{r}$ should be equal. Then we can get $r$ expressed in $\theta$ by equation (12):

$$
r=\frac{a_{2}-d_{20} \tan \alpha}{\sqrt[3]{\cos ^{3}(\theta-\alpha)+(1-\sin (\theta-\alpha))^{2}(2+\sin (\theta-\alpha)) \tan \alpha}}
$$

According to the geometrical relationship, we can also get the following formula:

$$
d_{2}=\frac{a_{2}-r \cos (\theta-\alpha)}{\tan \alpha}-h
$$

Then $d_{2}$ can be expressed as:

$$
d_{2}=\frac{a_{2}-r \tan \alpha(1-\sin (\theta-\alpha))-r \cos (\theta-\alpha)}{\tan \alpha}
$$

The liquid thickness and the radius of the interface are calculated according to formulae (12) and (14) by use of Matlab software. Figure 5 gives the details of the calculation, where $a_{2}=2 \mathrm{~mm}, d_{02}=0.5 \mathrm{~mm}, a=30^{\circ}$. In the figure, it is shown that the liquid thickness varies non-linearly with the radius. This will complicate the system optimization process.

Like a parallel plate, the thickness change of each liquid will cause spherical aberration and spherochromatism. Presuming that the initial thickness is the thickness when the interface is plane, the maximum spherical aberration

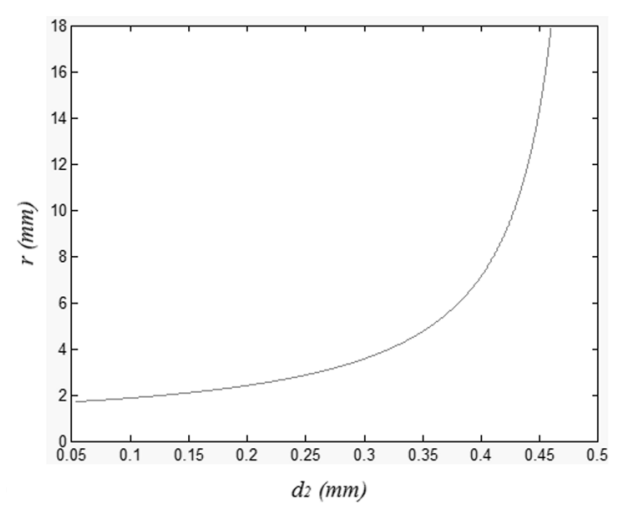

FIG. 5. Curve of thickness and the interface radius of the conical liquid lens, with the presumptions of a $2=2 \mathrm{~mm}, d_{02}=0.5 \mathrm{~mm}$, and $\mathrm{a}=30^{\circ}$. caused by the thickness change is as follow:

$$
\delta L=\frac{n_{1}-1}{2 n_{1}^{3}} u_{1}^{2} \Delta d-\frac{n_{2}-1}{2 n_{2}^{3}} u_{2}^{2} \Delta d
$$

As an illumination, for one type of Varioptic liquid lens, the maximum change of the thickness of each liquid is about $0.2 \mathrm{~mm}$. According to equation (15), the maximum spherical aberration caused by thickness change of one liquid lens is approximately $0.00024 u^{2}$. That means the thickness change should not be ignored during optical optimization, especially when the aperture angle is large and there are several pieces of liquid lens in the system.

\subsection{System Set Up and Optimized}

We use a commercial software (CODE V) to do the system design. The optimization is based on a damped least square (DLS) algorithm. Since the structure parameters (eg. thickness and interface radius) of the liquid lens are quite non-linear, the optimization process will be very complicated. Thanks for CODE V API (Application Programming Interface) we can realize the optimization process by using Microsoft Visual Basic (VB) to control the structure parameters of the liquid lens.

The CODE V API is an application programming interface designed to allow access from other programs to CODE $\mathrm{V}$ commands, which uses the Microsoft Windows standard Component Object Model (COM) interface. This enables us to execute CODE $\mathrm{V}$ commands using applications such as Microsoft Visual Basic (VB), Matlab, $\mathrm{C}++$ and so on. By use of this, the liquid lens can be modeled and optimized. Figure 6 shows the flow chart of the optimization process.

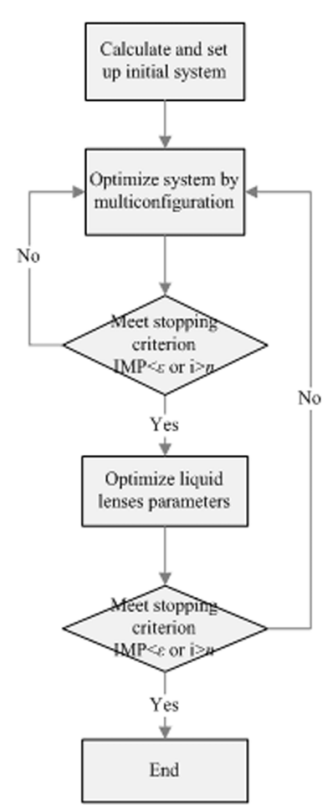

FIG. 6. Flow chart of the optimization process of the optical system contains liquid lenses. 
First, we calculate the initial structure and then optimize it by multi-configuration in CODE V. In this step, the thickness and interface radius of liquid lens are not strictly constrained. Second, we optimize the two liquid lenses for every zoom. The parameters of the two liquid lenses are strictly constrained and calculated by VB. And the parameters of the liquid lens are discretized for use during this optimization process. In this step, the optimization process may take a lot of time because of the need for multiple data exchanges between VB and CODE V. To enhance the feasibility, the parameters of the system are all frozen except the thickness and interface radius of the two liquid lenses. A stopping criterion which consists of max loops number and improvement (IMP) of error function (EF) is set up before the optimization,. The optimization process is based on damped least square (DLS) algorithm. The error function $(\mathrm{EF})$ is defined as follows:

$$
\begin{aligned}
& E F(n)=\sum_{z=1}^{m} \sum_{k=1}^{k} w_{k}\left|S P O_{z}^{k}\right|+w_{k+1} \sum_{z=1}^{m}\left|A X_{z}-A X_{z}^{0}\right|+w_{k+2} \sum_{z=1}^{m}\left|L A T_{z}-L A T_{z}^{0}\right| \\
& +w_{k+3} \sum_{z=1}^{m}\left|E F L_{z}-E F L_{z}^{0}\right|+w_{k+4} \sum_{z=1}^{m}\left|D I S_{z}-D I S_{z}^{0}\right|
\end{aligned}
$$

where $w_{l} \sim w_{k+1}$ are weights to tune the fitness value for various situations, $z$ is the number of the zoom, $k$ is the number of Field of View (FOV), $S P O{ }_{z}^{k}$ is the spot size at each field $k$ of each zoom $z, A X_{z}, L A T_{z}, E F L_{z}, D I S_{z}$ are the simulation of axial chromatic aberration, lateral chromatic aberration, effective focal length and distortion at each zoom, $A X_{z}^{0}, L A T_{z}^{0}, E F L_{z}^{0}, D I S_{z}^{0}$ are their thresholds.

\section{DESIGN EXAMPLE}

A two liquid lens zoom system is designed in this section. The long focal length of the system is $f_{L}^{\prime}=8.6 \mathrm{~mm}$, while the short focal length of the system is $f_{S}{ }^{\prime}=4.8 \mathrm{~mm}$. The image height is $1.745 \mathrm{~mm}$. The overall length of the system is $10 \mathrm{~mm}$. And the zoom factor is 1.8 .

The liquid lens parameters are based on Varioptic liquid lens ARCTIC 416. The structure of the lens is shown in Figure 7 (the material of glass1 is same as that of glass2). Lens details can be seen from www.varioptic.com. The lens data of the liquid lens thickness and radius are discretized for use.

The specification of the system is shown in Table 1 .

The initial system structure is calculated according to

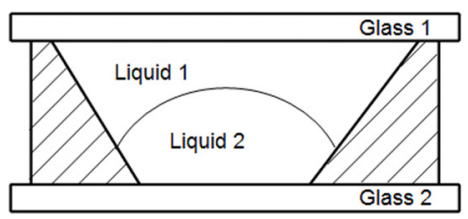

FIG. 7. Schematic drawing of Varioptic liquid lens.

\begin{tabular}{|c|c|c|c|c|c|}
\hline \multicolumn{6}{|c|}{ Specification } \\
\hline & Zoom1 & Zoom2 & Zoom3 & Zoom4 & Zoom5 \\
\hline EFL (mm) & 8.6 & 7.7 & 6.7 & 5.7 & 4.8 \\
\hline Image height $(\mathrm{mm})$ & \multicolumn{5}{|c|}{1.745} \\
\hline $\mathrm{BEF}(\mathrm{mm})$ & \multicolumn{5}{|c|}{0.4} \\
\hline OAL (mm) & \multicolumn{5}{|c|}{10} \\
\hline $\mathrm{f} / \#$ & 5.4 & 4.8 & 4.2 & 3.6 & 3.2 \\
\hline \multirow{2}{*}{ Distortion } & $-6.1 \%$ & $-4.9 \%$ & $-1.8 \%$ & $-5.4 \%$ & $-6.5 \%$ \\
\hline & $-7.9 \%$ & $-5.9 \%$ & $-3.4 \%$ & $-6.8 \%$ & $-8.3 \%$ \\
\hline $\begin{array}{c}\text { MTF }(100 \% \text { FOV, } \\
401 \mathrm{p} / \mathrm{mm})\end{array}$ & 0.28 & 0.38 & 0.41 & 0.35 & 0.27 \\
\hline \multirow{2}{*}{$\begin{array}{l}\text { Sim-aperture of } \\
\text { lens }(\mathrm{mm})\end{array}$} & $\max$ & \multicolumn{4}{|c|}{2.15} \\
\hline & $\min$ & \multicolumn{4}{|c|}{0.49} \\
\hline
\end{tabular}

TABLE 1. Specification of the two liquid lens zoom system

\begin{tabular}{|c|c|c|c|}
\hline Surface\# & Curvature radius & Thickness & Glass \\
\hline Object & Infinity & Infinity & \\
\hline 1 & (A) 5.4601 & 0.640 & 631591.543590 \\
\hline 2 & -16.0625 & 0.9189 & \\
\hline 3 & -6.4981 & 0.2588 & 694941.305099 \\
\hline 4 & 25.2493 & 0.5584 & \\
\hline \multirow{4}{*}{$\begin{array}{l}\text { Liquid } \\
\text { lens } 1\end{array}$} & Infinity & 0.3 & 523100,55000 \\
\hline & Infinity & zoom & 488900,39500 \\
\hline & zoom & zoom & 399900,60600 \\
\hline & Infinity & 0.55 & 523100,55000 \\
\hline 9 & Infinity & 0.9241 & \\
\hline stop & Infinity & 0.4808 & \\
\hline 11 & 8.3777 & 0.7468 & 599839.614300 \\
\hline 12 & -4.9992 & 0.2523 & \\
\hline \multirow{4}{*}{$\begin{array}{l}\text { Liquid } \\
\text { lens } 2\end{array}$} & Infinity & 0.3 & 523100,55000 \\
\hline & Infinity & zoom & 488900,39500 \\
\hline & zoom & zoom & 399900,60600 \\
\hline & Infinity & 0.55 & 523100,55000 \\
\hline 17 & Infinity & 2.3708 & \\
\hline 18 & 118.7351 & 0.1 & 596957.390434 \\
\hline 19 & 4.4421 & 0.4059 & \\
\hline Image & Infinity & 0.00 & \\
\hline
\end{tabular}

TABLE 2. General parameters of the system

TABLE 3. Zoom parameters of the system

\begin{tabular}{c|c|c|c|c|c|c}
\hline \hline \multirow{2}{*}{$\begin{array}{c}\text { Zoom } \\
\text { position }\end{array}$} & \multicolumn{2}{|c|}{$\begin{array}{c}\text { Curvature radius } \\
(\mathrm{mm})\end{array}$} & \multicolumn{4}{c}{ Thickness (mm) } \\
\cline { 2 - 7 } & $\mathrm{S} 7$ & $\mathrm{~S} 16$ & $\mathrm{~S} 6$ & $\mathrm{~S} 7$ & $\mathrm{~S} 15$ & $\mathrm{~S} 16$ \\
\hline 1 & 12.12 & -3.64 & 0.374 & 0.276 & 0.200 & 0.400 \\
\hline 2 & -44.10 & -4.50 & 0.321 & 0.329 & 0.208 & 0.442 \\
\hline 3 & -8.01 & -10.83 & 0.259 & 0.391 & 0.275 & 0.375 \\
\hline 4 & -5.24 & 25.82 & 0.224 & 0.426 & 0.306 & 0.344 \\
\hline 5 & -3.86 & 5.08 & 0.200 & 0.400 & 0.222 & 0.428 \\
\hline
\end{tabular}



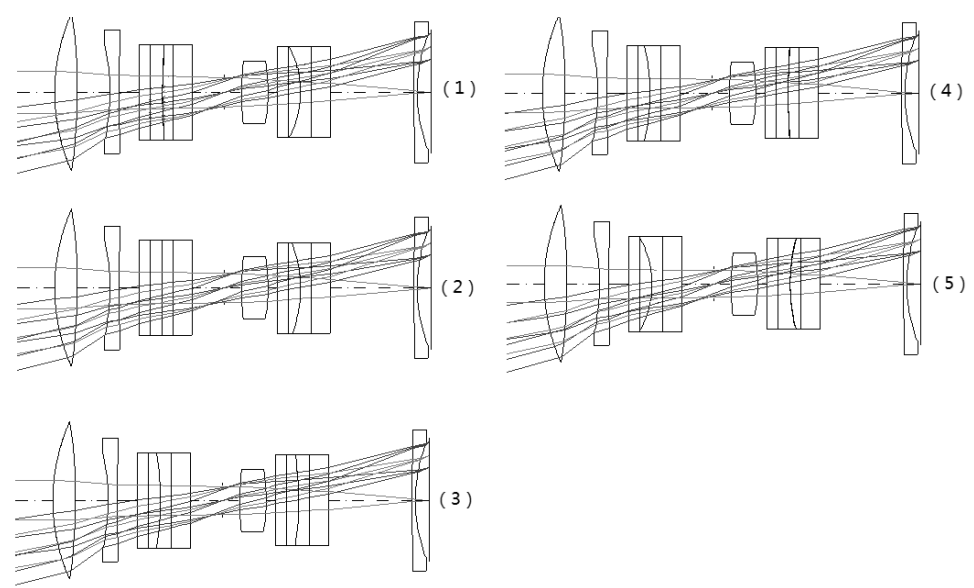

FIG. 8. Layout of the system at different zoom positions.

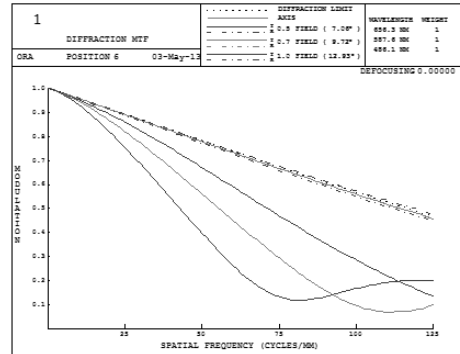

(1)

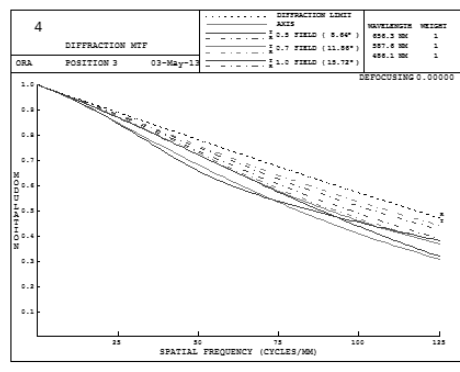

(4)

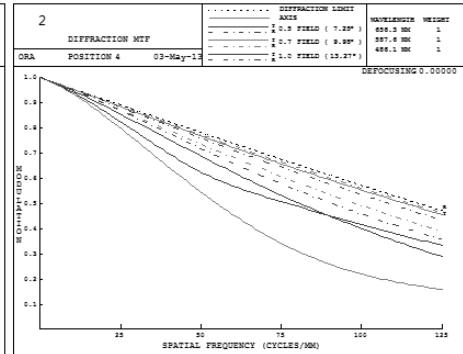

(2)

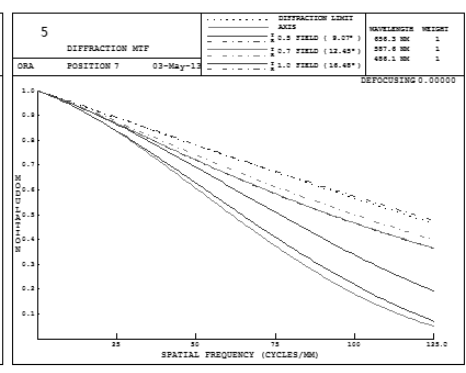

(5)

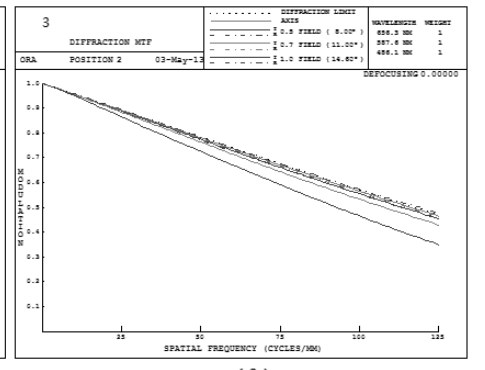

(3)

FIG. 9. Curve of MTF (Modulation transform function) of each zoom position.

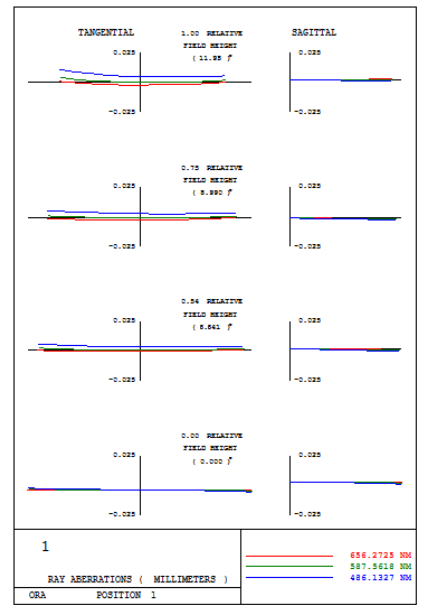

(1)

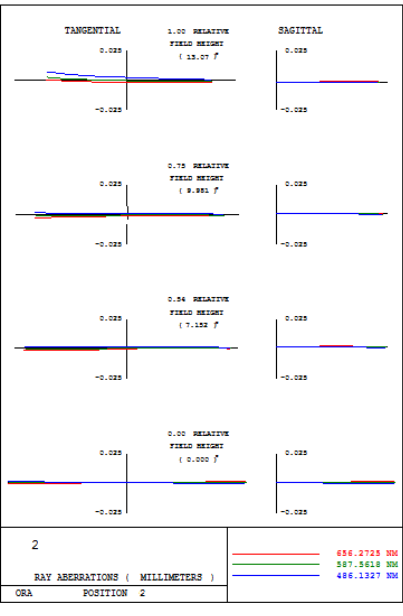

(2)

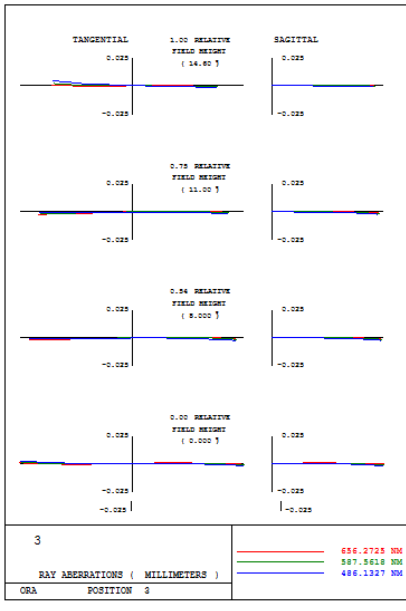

(3)

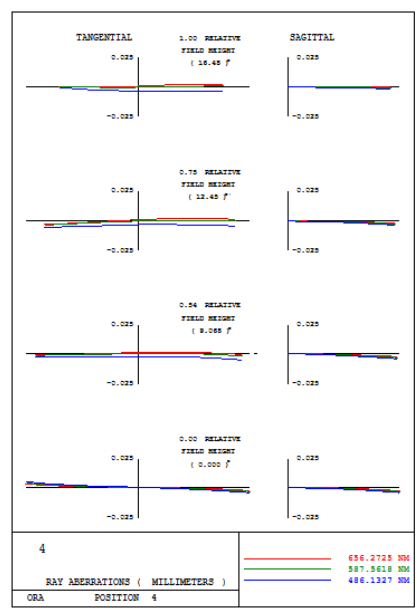

(4)

FIG. 10. Ray aberration curves of the designed system of each zoom position. 
Section 2. To realize the system, three groups of classical lenses are adopted to eliminate the aberrations. The first group of solid lenses is in front of the system, which is used to eliminate the incident angle of the chief rays. The first surface of the first lens is set aspherical to balance the off-axis aberration. The second solid lens is put behind the aperture to bear part of the optical power. The third group of solid lenses with slight negative power is behind the second liquid lens, which is set to eliminate the aberration when the liquid lens has a strong positive.

The general parameters of the system are shown in Table 2.

The zoom parameters of the system are shown in Table 3.

The layout of the system at different zoom positions is shown in Fig. 8.

The Fig. 9, 10 show the imaging performance of the system.

\section{SUMMARY}

A liquid lens can help a zoom system become more miniature than before due to its character of active zooming. This has a very wide potential application in many areas. In this paper, we try to give a method for designing and optimizing a zoom system including liquid lenses. First the zoom equation of the system composed of two liquid lenses is deduced. Then the structure parameters of the conical double-liquid electrowetting based lens are analyzed and calculated by Matlab software. It is shown that the liquid thickness varies quite nonlinearly with the interface radius in a liquid lens. Through the API of the commercial optical design software CODE V, the zoom system with liquid lenses is modeled and optimized successfully. The optimization process flow chart is given. The structure parameters of the liquid lens is strictly constrained and calculated by VB during the optimization. A zoom system is designed for illustration, in which two pieces of liquid lens and four pieces of traditional lens are used and a zoom factor of 1.8 is implemented. The overall length of the system is $10 \mathrm{~mm}$. This has potential application in mobile phone cameras or tablet cameras.

\section{ACKNOWLEDGMENT}

This work is supported by the National Natural Science Foundation of China (Grant No. 61205015), the Research Fund for the Doctoral Program of Higher Education of China (20123120130001), and is partly supported by the Leading Academic Discipline Project of Shanghai Municipal Government (S30502) and the National Natural Science Foundation of China (Grant No. 61108051).

\section{REFERENCES}

1. M. Amberg, A. Oeder, A. Sinzinger, P. J. W. Hands, and G. D. Love, "Tuneable planar integrated optical systems," Opt. Express 15, 10607-10614 (2007).

2. J. Li. G. Baird, Y. H. Lin, H. Ren, and S. T. Wu, "Refractive-index matching between liquid crystals and photopolymers," Soc. Info. Display 13, 1017-1026 (2005).

3. A. H. Robert and B. J. Feenstra, "Video-speed electronic paper based on electrowetting," Nature 25, 383-385 (2003).

4. S. Kuiper and B. H. W. Hendriks, "Variable-focus lens for miniature cameras," Appl. Phys. Lett. 85, 100-109 (2004).

5. B. H. W. Hendriks, S. Kuiper, M. A. J. van As, C. A. Renders, and T. W. Tukker, "Variable liquid lenses for electronic products," Proc. SPIE 6034, 603402 (2006).

6. H. Ren and S.-T. Wu, "Variable-focus liquid lens by changing aperture," Appl. Phys. Lett. 86, 211107 (2005).

7. S. W. Lee and S. S. Lee, "Focal tunable liquid lens integrated with an electromagnetic actuator," Appl. Phys. Lett. 90, 121129 (2007).

8. J.-K. Lee, K.-W. Park, G.-B. Lim, H.-R. Kim, and S.-H. Kong, "Variable-focus liquid lens based on a laterallyintegrated thermopneumatic actuator," J. Opt. Soc. Korea 16, 22-28 (2012).

9. W. Zhang and W. Tian, "A novel micro zoom system design with liquid lens," Proc. SPIE 7156, 715603 (2009).

10. A. Miks and J. Novak, "Analysis of two-element zoom systems based on variable power lenses," Opt. Express 18, 6797-6810 (2010).

11. J. Sun, B. Hsueh, Y. Fang, J. MacDonald, and C. Hu, "Optical design and multiobjective optimization of miniature zoom optics with liquid lens element," Appl. Opt. 48, 1741-1757 (2009).

12. S.-C. Park and W.-S. Lee, "Design and analysis of a one-moving-group zoom system using a liquid lens," J. Korean Phys. Soc. 62, 435-442 (2013).

13. Y.-C. Fang, C.-M. Tsai, and C.-L. Chung, "A study of optical design and optimization of zoom optics with liquid lenses through modified genetic algorithm," Opt. Express 19, 16291-16302 (2011).

14. R. Peng, J. Chen, and S. Zhuang, "Electrowetting-actuated zoom lens with sphericalinterface liquid lenses," J. Opt. Soc. Am. A 25, 2644-2650 (2008).

15. R. Peng, J. Chen, Ch. Zhu, and S. Zhuang, "Design of a zoom lens without motorized optical elements," Opt. Express 15, 6664-6669 (2007).

16. Y. Fang and C. Tsai, "Miniature lens design and optimization with liquid lens element via genetic algorithm," J. Opt. A: Pure Appl. Opt. 10, 075304 (2008).

17. Z. Wang, Y. Xu, and Y. Zhao, "Aberration analyses of liquid zooming lenses without moving parts," Opt. Commun. 275, 22-26 (2007).

18. A. Miks, J. Novak, and P. Novak, "Generalized refractive tunable-focus lens and its imaging characteristics," Opt. Express 18, 9034-9047 (2010).

19. M. Ye, M. Noguchi, B. Wang, and S. Sato, "Zoom lens system without moving elements realized using liquid crystal lenses," Electron. Lett. 45, 646-648 (2009). 